\title{
Investigation of miRNA- and IncRNA-mediated competing endogenous RNA network in cholangiocarcinoma
}

\author{
YANXIN HE ${ }^{1}, \mathrm{CHAO} \mathrm{LIU}^{1}$, PAN SONG $^{2}$, ZHIGANG PANG $^{1}$, ZHUOMAO MO $^{3}$, \\ CHUIGUO HUANG $^{4}$, TINGTING YAN ${ }^{5}$, MENG SUN $^{6}$ and XIANEN FA ${ }^{1}$
}

\begin{abstract}
${ }^{1}$ Department of Surgery, The Second Affiliated Hospital of Zhengzhou University; ${ }^{2}$ Department of Urology, The First Affiliated Hospital of Zhengzhou University, Zhengzhou, Henan 450014; ${ }^{3}$ College of Traditional Chinese Medicine of Jinan University, Institute of Integrated Traditional Chinese and Western Medicine of Jinan University, Guangzhou, Guangdong 510632; ${ }^{4}$ Department of Urology, The Second Affiliated Hospital of Zhengzhou University, Zhengzhou, Henan 450014; ${ }^{5}$ The Nethersole School of Nursing, Faculty of Medicine, The Chinese University of Hong Kong, Hong Kong 999077; ${ }^{6}$ Department of Gynecology and Obstetrics, The Second Affiliated Hospital of Zhengzhou University, Zhengzhou, Henan 450014, P.R. China
\end{abstract}

Received August 29, 2018; Accepted March 8, 2019

DOI: $10.3892 / 01.2019 .10852$

\begin{abstract}
Cholangiocarcinoma (CCA) is a biliary malignancy which is prone to lymphatic metastasis and has a high mortality rate. This disease lacks effective therapeutic targets and prognostic molecular biomarkers. The aim of the current study was to investigate differentially expressed genes and elucidate their association with CCA and the underlying mechanisms of action. mRNAs, long non-coding RNAs (lncRNAs) and microRNAs (miRNAs) obtained from 36 CCA samples and nine normal samples from The Cancer Genome Atlas were integrated. Subsequently, 1,095 differentially expressed (DE) mRNAs and 75 DE miRNAs were identified using a threshold of $\mid \log 2$ fold changel $>2$ and an adjusted $\mathrm{P}<0.01$. Weighted gene co-expression network analysis was used to identify the DEmRNAs that could be key target genes in CCA. A total of 12 hub DEmRNAs were identified as targetable genes. Furthermore, the hub DEmRNAs-DElncRNAs pairs were identified using the miRTarBase and miRcode databases. Cytoscape software was used to construct and visualize the protein-protein interactions and the competing endogenous RNA network. Survival time analysis and correlation analysis were used to
\end{abstract}

Correspondence to: Dr Xianen Fa, Department of Surgery, The Second Affiliated Hospital of Zhengzhou University, 2 Jingba Road, Zhengzhou, Henan 450014, P.R. China

E-mail: faxezzu@163.com

Dr Chuiguo Huang, Department of Urology, The Second Affiliated Hospital of Zhengzhou University, 2 Jingba Road, Zhengzhou, Henan 450014, P.R. China

E-mail: huangcg0727@163.com

Key words: cholangiocarcinoma, weighted gene co-expression network analysis, competing endogenous RNA network, survival prognosis further evaluate the hub genes. The results obtained in the current study suggested that spalt like transcription factor 3 and OPCML intronic transcript 1 may serve an important role in the development and progression of CCA.

\section{Introduction}

Cholangiocarcinoma (CCA) is a biliary malignancy which originates in the bile duct epithelium and can be subclassified according to the location as intrahepatic, extrahepatic or perihilar (1-3). Although rare, the incidence of CCA has increased in the past decade $(2,3)$. CCA is prone to lymphatic metastasis and has a high mortality rate. CCA may be difficult to diagnose until advanced stages of the disease. The majority of patients with CCA are diagnosed at an advanced stage of the disease, when loco-regional invasion or distant metastasis has occurred $(2,3)$. Curative surgical intervention for patients in an early stage is unlikely (2). Although the diagnostic methods for CCA have improved, several patients with CCA face delayed or inaccurate diagnoses, resulting in a decreased five-year overall survival time (3). This suggests that novel biomarkers for the early diagnosis of CCA as well as specific targeted therapy are required to improve patient outcomes in CCA.

Next generation RNA sequencing technology has revealed the complexity and diversity of the human genome (4). Non-coding RNAs (ncRNAs) constitute the majority of the transcriptome and include microRNAs (miRNAs), long non-coding RNAs (lncRNAs) and circle-RNAs (4-6). ncRNAs are involved in several important biological functions, such as RNA transcription and intracellular and intercellular communication (4). Furthermore, lncRNAs may serve as novel biomarkers and drug targets, in different types of cancer, including renal, breast and prostate cancer $(5,6)$. However, the mechanism by which lncRNAs regulate the expression of genes remains unclear.

Salmena et al (7) proposed the competing endogenous RNA (ceRNA) hypothesis, which suggest that lncRNAs may act as 
ceRNAs to suppress miRNA function by sharing one or more miRNA response elements. This hypothesis has since been supported by a number of studies $(8,9)$. IncRNAs may modulate genes at the protein-coding level and take part in the regulation of cellular processes by competing with miRNAs $(10,11)$. The importance of the IncRNA-miRNA-mRNA regulatory network in tumor pathogenesis and progression has been demonstrated in numerous studies (12-14). However, the role of the ceRNA network in CCA has not been elucidated and requires further investigation.

Weighted gene co-expression network analysis (WGCNA) is a novel systems biology method for describing the correlation patterns among genes across microarray samples $(15,16)$. WGCNA may be used to identify modules of highly related genes, to summarize such clusters using eigengenes or intramodular hub genes, to associate modules to one another and to external sample traits, and to calculate module membership measures $(17,18)$. Correlation networks may be applied to network-based gene screening methods that can be used to identify candidate biomarkers or therapeutic targets (17-19). The present study performed WGCNA and multiple database analyses to construct a ceRNA network to elucidate the interactions between differentially expressed (DE) lncRNAs, DEmiRNAs and DEmRNAs in CCA.

\section{Materials and methods}

Study population. A total of 45 CCA samples were used in a comprehensive integrated analysis, including 36 samples from patients and 9 from healthy controls. The dataset was downloaded from the The Cancer Genome Atlas database (TCGA; cancergenome.nih.gov) using the Genomics Data Commons Data Transfer Tool, release number 16.0 (gdc.cancer. gov/access-data/gdc-data-transfer-tool). The data included a level three gene expression file of mRNAseq and miRNAseq data as well as patient clinical information. The current study met the publication guidelines provided by TCGA (cancergenome.nih.gov/publications/publicationguidelines).

Differential expression analysis. The mRNAseq data used in the current study were derived from 45 samples, including 36 CCA samples (tumor cohort) and 9 normal tissue samples that were extracted from adjacent tissues (normal cohort). The data from the two types of samples were merged and the data points that were close to zero were removed. The 'DESeq' package (www.bioconductor.org/packages/DESeq) in R software version 3.5.2 (www.r-project.org) was used to identify the DEmRNAs and DEmiRNAs. A threshold of llog2 fold change $(\mathrm{FC}) \mid>2.0$ and an adjusted $\mathrm{P}<0.01$ were used to select the DEmRNAs and DEmiRNAs. The Encyclopedia of DNA Elements (www.encodeproject.org) was used to define and annotate the IncRNAs. The DElncRNAs with cut-off criteria of $\log 2 \mathrm{FCl}>2.0$ and an adjusted $\mathrm{P}<0.01$ were selected. To remove any potential noise, the FC values associated with the comparisons that were not considered significant by the 'limma' package (www.bioconductor.org/packages/limma) or potentially significant by threshold-filtering were converted to 'zero', where the $\log 2$ scale corresponded with the complete absence of differential regulation among all samples. In addition, the GeneCards database (https://www.genecards.
Table I. Clinical characteristics of the 36 patients with cholangiocarcinoma.

\begin{tabular}{lc} 
Variables & Patient characteristics \\
\hline Age, mean years \pm SD & $63.48 \pm 12.85$ \\
Overall survival time, & $25.49 \pm 18.56$ \\
mean months \pm SD & \\
Sex, n (\%) & \\
Male & $16(44.4)$ \\
Female & $20(55.6)$ \\
Ethnicity, n $(\%)$ & \\
Caucasian & $31(86.2)$ \\
Asian & $3(8.3)$ \\
African American & $2(5.5)$ \\
Tumor stage, n $(\%)$ & \\
Stage I & $19(52.7)$ \\
Stage II & $9(25.0)$ \\
Stage III & $1(2.8)$ \\
Stage IV & $2(5.6)$ \\
Stage IVa & $2(5.6)$ \\
Stage IVb & $3(8.3)$ \\
Alive, n $(\%)$ & \\
Yes & $18(50.0)$ \\
No & $18(50.0)$ \\
\hline
\end{tabular}

org/) was used for annotation, as it is a searchable, integrative database that provides comprehensive information on all annotated human genes.

Weighted gene co-expression network construction and functional annotation. WGCNA, a systematic biology method, describes the co-expression patterns between genes across microarray samples. WGCNA can be used for identifying modules of highly correlated genes, for summarizing these clusters using the module eigengene or an intramodular hub gene, for relating modules to one another and to external sample traits, and for calculating module membership measures. Co-expression networks facilitate network-based gene screening methods that can be used to identify candidate biomarkers or therapeutic targets (18). Therefore, to identify the interactions between the differentially expressed genes (DEGs), the 'WGCNA' package (www.bioconductor. org/packages/WGCNA) in R software was used to identify co-expression modules, with a power cut-off threshold of four and a minimum module size limited to 20 in the present study.

In order to confirm Kyoto Encyclopedia of Genes and Genomes (KEGG; genome.jp/kegg) pathways of DEGs, the 'cluster profiler' package (www.bioconductor.org/packages/clusterprofiler) in R software was used to annotate the function of the different module genes. The Search Tool for the Retrieval of Interacting Genes (STRING; www.string-db. org) was subsequently used to evaluate the protein-protein interaction (PPI) network for the different colored modules, and Cytoscape software 3.2.0 (https://cytoscape.org/) was used to construct the PPI network. 


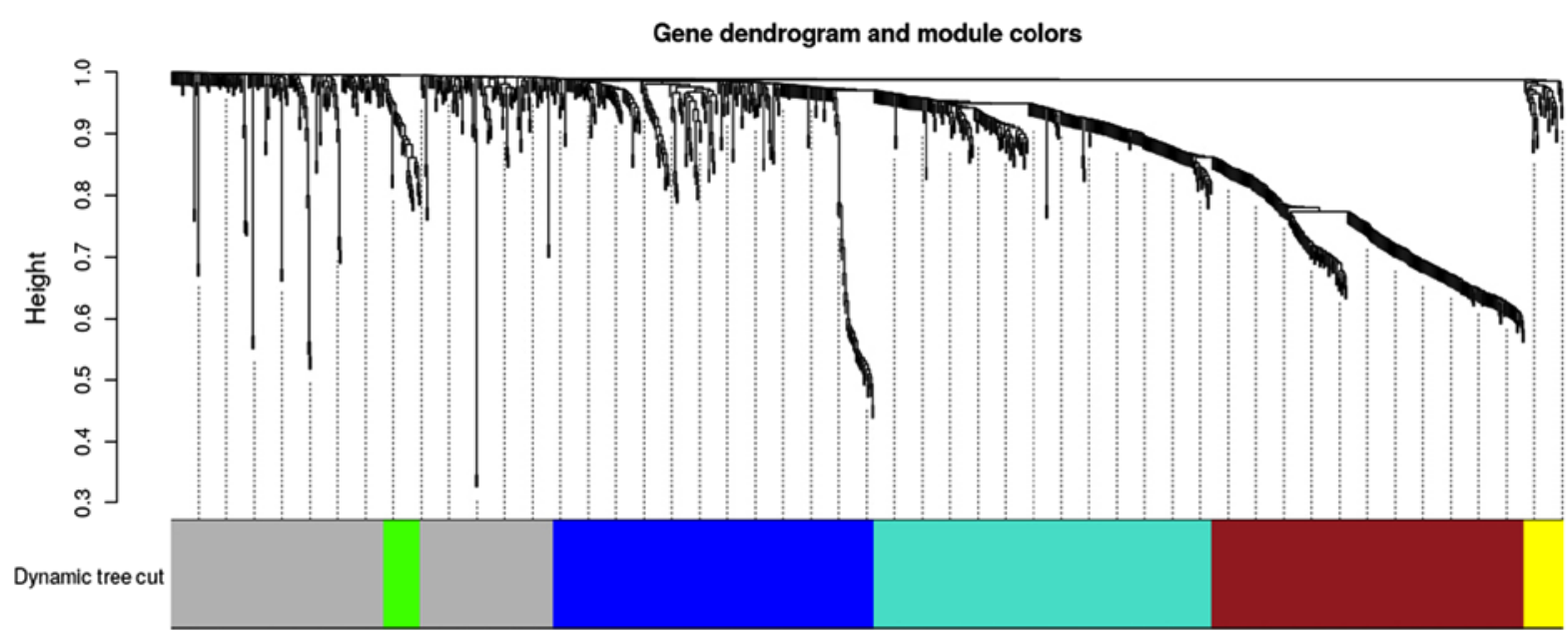

Figure 1. Hierarchical clustering dendrogram of identified coexpressed genes in modules. This dendrogram was generated by unsupervised hierarchical clustering of genes using topological overlap. The colored strips below each dendrogram demostrate the module designation that was identified through the clusters of coexpressed genes.

Construction of the ceRNA network. To investigate the association between IncRNA and miRNA in the ceRNA network, a coexpression network of DEmRNA, DEmiRNA and DElncRNA and was constructed using Cytoscape software. The pairs of miRNA-mRNA were selected using miRTarBase (http://mirtarbase.mbc.nctu.edu.tw) (20). The data in miRTarBase has been verified by various methods, including the reporter assay, reverse transcription quantitative PCR, western blotting, microarray and next-generation sequencing experiments. Furthermore, the lncRNA-miRNA interactions were assessed using the miRcode database (www.mircode.org), which includes $>10,000$ lncRNA genes (21).

Survival and co-expression analysis. The clinical data of patients with CCA were used to identify the prognostic DE RNA signatures and a multivariate Cox proportional hazard regression analysis was performed. The regression coefficient of each gene was determined using the 'survival' package (www.bioconductor.org/packages/surival) in R software. The coefficient of each selected gene presented the estimated logarithm of the hazard ratio. The risk score formula was used to define the high- and low-risk groups. The Kaplan-Meier method was used to assess the survival time difference between two groups using the log-rank test. $\mathrm{P}<0.05$ was considered to indicate a statistically significant difference.

Pearson's correlation coefficient analysis. The Pearson's correlation coefficient was used to identify the correlations between DElncRNAs and DEmRNAs involved in the established ceRNA network. $\mathrm{P}<0.01$ and $\mathrm{R}>0.5$ were used to indicate a statistically significant difference.

\section{Results}

Identification of DEmRNAs, DEmiRNAs and DElncRNAs. The clinical information of the patients from whom the 36 CCA samples were obtained is presented in Table I. Using the 'DESeq' package in R software, 1,095 DEmRNAs and 75 DEmiRNAs were identified. The results obtained in the current study indicated that there were $862(78.72 \%)$ upregulated and 233 (21.28\%) downregulated DEmRNAs. In addition, a total of 70 upregulated and 5 downregulated DEmiRNAs were identified. Furthermore, a total of 485 aberrantly expressed lncRNAs in CCA tissues compared with normal tissues were identified using the GeneCards database The heatmap clustering of DEmRNAs, DEmiRNAs and DElncRNA is presented in Figs. S1-3.

Construction of weighted gene co-expression modules. In order to identify the functional clusters in patients with CCA, the gene co-expression network analysis of 1,095 DEmRNAs was performed by WGCNA. As illustrated in Fig. 1, six color modules were clustered, while the non-clustering DEmRNAs were presented in grey. Each branch in the dendrogram signified a single gene. The area occupied by each color illustrated the number of genes within the corresponding module.

PPI and functional annotation for the modules. Using the STRING tool, 4 PPI networks were identified in the modules, including turquoise, blue, brown and yellow modules. However, a relevant PPI was not identified in the green module. Additionally, in order to identify the function and PPI of DEmRNAs in the different colored modules, KEGG pathway annotation for each module was performed and is presented in Table II. Hub DEmRNAs from the PPI networks were selected for ceRNA network construction. As illustrated in Fig. 2, the relevant modules (turquoise, blue, brown and yellow) were selected for visualization of the Cytoscape software results.

Construction of the ceRNA network in CCA. To further explore the association between IncRNAs and miRNAs in patients with CCA, a ceRNA network was constructed using the three types of DE RNAs. DEmiRNA-mRNA pairs were predicted using the miRTarBase database. A total of 12 hub DEmRNAs were identified as targeted genes by WGCNA. These were LRRK2, TBC1D2, E2F1, SHISA6, CYP1B1, TMEM100, PCDHAC2, GRIK3,FOXQ1, PCDHA1, HOXC13 and SALL3.DEmiRNAs, including hsa-mir-519d, hsa-mir-31, hsa-mir-506, hsa-mir-372, 
Table II. KEGG pathway analysis of weighted gene co-expression network analysis modules.

A, Turquoise

\begin{tabular}{|c|c|c|c|}
\hline KEGG pathway & Input number & Top genes & P-value \\
\hline 'Regulation of signaling' & 76 & SHISA6, PCDHA1, SALL3, & $8.5 \times 10^{-05}$ \\
\hline 'Regulation of cell communication' & 73 & CYP1B1, SERPINA1, TIMP1, & $3.2 \times 10^{-04}$ \\
\hline 'Regulation of multicellular organismal process' & 68 & CD177, PRSS3 & $4.6 \times 10^{-05}$ \\
\hline 'Anatomical structure morphogenesis' & 59 & & $1.8 \times 10^{-04}$ \\
\hline 'Tissue development' & 47 & & $9.2 \times 10^{-04}$ \\
\hline ‘Secretion’ & 46 & & $8.0 \times 10^{-05}$ \\
\hline 'Cell-cell signaling' & 45 & & $8.5 \times 10^{-05}$ \\
\hline 'Secretion by cell' & 45 & & $4.6 \times 10^{-05}$ \\
\hline 'Positive regulation of cell communication' & 43 & & $8.6 \times 10^{-04}$ \\
\hline 'Positive regulation of multicellular organismal process' & 41 & & $7.9 \times 10^{-04}$ \\
\hline 'Neuron differentiation' & 35 & & $9.2 \times 10^{-04}$ \\
\hline 'Regulation of secretion' & 25 & & $9.2 \times 10^{-04}$ \\
\hline 'Regulation of secretion by cell' & 24 & & $9.2 \times 10^{-04}$ \\
\hline 'Epidermis development' & 19 & & $1.3 \times 10^{-03}$ \\
\hline
\end{tabular}

B, Blue

\begin{tabular}{lcc}
\hline KEGG pathway & Input number & Top genes \\
\hline 'Cellular developmental process' & 93 & GRIK3, E2F1, PCDHAC2, \\
'System development' & 92 & MMP3, PAX7, S100B \\
'Cell differentiation' & 91 & $6.4 \times 10^{-08}$ \\
'Anatomical structure morphogenesis' & 53 & $4.1 \times 10^{-06}$ \\
'Response to external stimulus' & 48 & $6.4 \times 10^{-08}$ \\
'Tissue development' & 46 & $9.9 \times 10^{-04}$ \\
'Cell adhesion' & 38 & $9.9 \times 10^{-04}$ \\
'Chemotaxis' & 22 & $3.7 \times 10^{-04}$ \\
'Extracellular structure organization' & 22 & $2.2 \times 10^{-04}$ \\
'Extracellular matrix organization' & 21 & $2.2 \times 10^{-04}$ \\
'Cell chemotaxis' & 14 & $1.1 \times 10^{-06}$ \\
'Collagen metabolic process' & 12 & $4.0 \times 10^{-07}$ \\
'Collagen catabolic process' & 10 & $6.6 \times 10^{-04}$ \\
\hline
\end{tabular}

C, Brown

\begin{tabular}{lcrr}
\hline KEGG pathway & Input number & \multicolumn{1}{c}{ Top genes } \\
\hline 'Nervous system development' & 49 & FOXQ1, HOXC13, TMEM100, & $7.2 \times 10^{-04}$ \\
'Cell-cell signaling' & 43 & PAX7, COL13A1, NPHS1, \\
'Transmembrane transport' & 41 & MXRA8 & $8.6 \times 10^{-05}$ \\
'Behavior' & 24 & \\
'Chemical synaptic transmission' & 23 & $6.1 \times 10^{-05}$ \\
'Ossification' & 16 & $1.5 \times 10^{-04}$ \\
'Learning or memory' & 15 & $6.3 \times 10^{-04}$ \\
'Neurotransmitter transport' & 13 & $8.6 \times 10^{-05}$ \\
'Memory' & 9 & $5.9 \times 10^{-04}$ \\
'Hippocampus development' & 8 & $5.9 \times 10^{-04}$ \\
'Positive regulation of fat cell proliferation' & 3 & $4.0 \times 10^{-04}$ \\
& &
\end{tabular}


Table II. Continued.

D, Yellow

\begin{tabular}{lcrr} 
KEGG pathways & Input number & Top genes & P-value \\
\hline 'Cell morphogenesis' & 7 & TBC1D2, LRRK2 & $9.9 \times 10^{-03}$ \\
'Cell part morphogenesis' & 6 & $9.4 \times 10^{-03}$ \\
'Tangential migration from the subventricular & 2 & $9.4 \times 10^{-03}$
\end{tabular}

zone to the olfactory bulb'

KEGG, Kyoto Encyclopedia of Genes and Genomes; SHISA6, shisa family member 6; PCDHA1, protocadherin $\alpha$ 1; SALL3, spalt like transcription factor 3; CYP1B1, cytochrome P450 family 1 subfamily B member 1; SERPINA1, serpin family A member 1; TIMP1, TIMP metallopeptidase inhibitor 1; CD177, CD177 molecule; PRSS3, serine protease 3; GRIK3, glutamate ionotropic receptor kainate type subunit 3; E2F1, E2F transcription factor 1; PCDHAC2, protocadherin $\alpha$ subfamily C, 2; MMP3, matrix metallopeptidase 3; PAX7, paired box 7; S100B, S100 calcium binding protein B; FOXQ1, forkhead box Q1; HOXC13, homeobox C13; TMEM100, transmembrane protein 100; COL13A1, collagen type XIII $\alpha 1$ chain; NPHS1, NPHS1 adhesion molecule, nephrin; MXRA8, matrix remodeling associated 8; TBC1D2, TBC1 domain family member 2; LRRK2, leucine rich repeat kinase 2 .

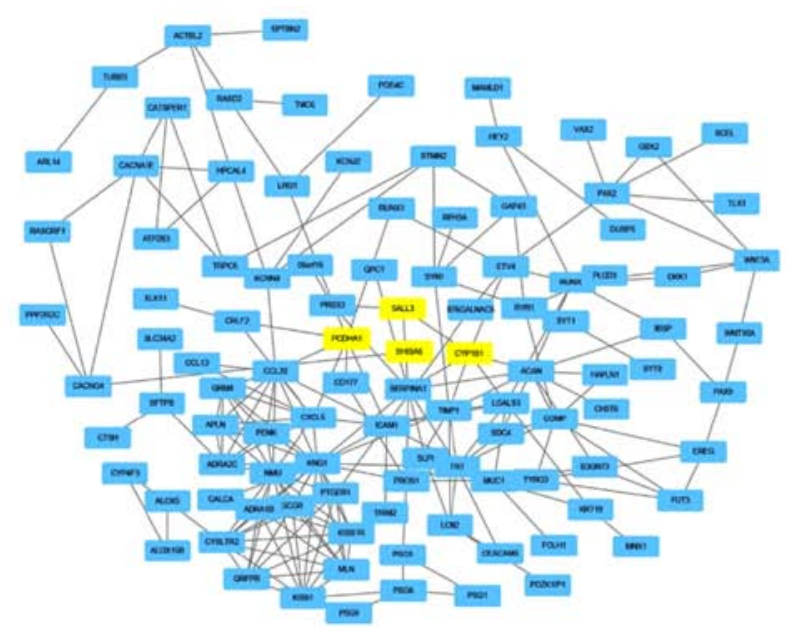

Turquoise module

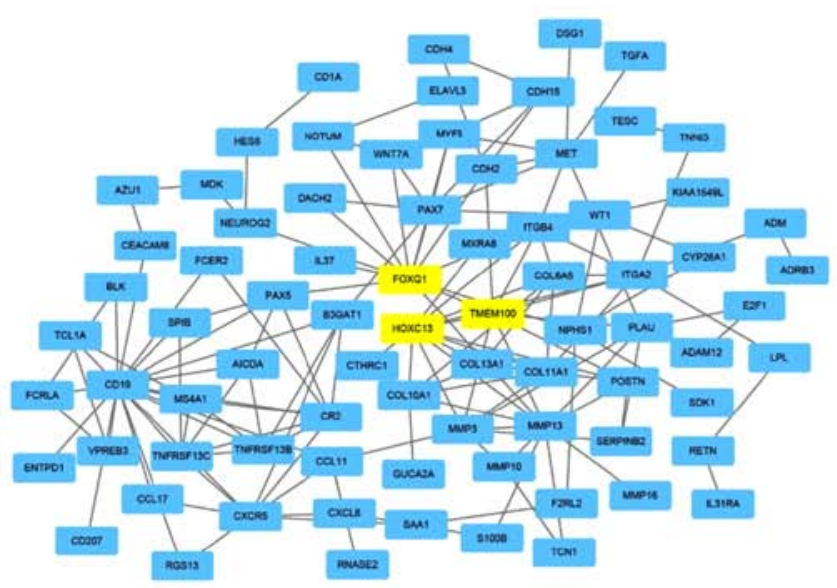

Brown module

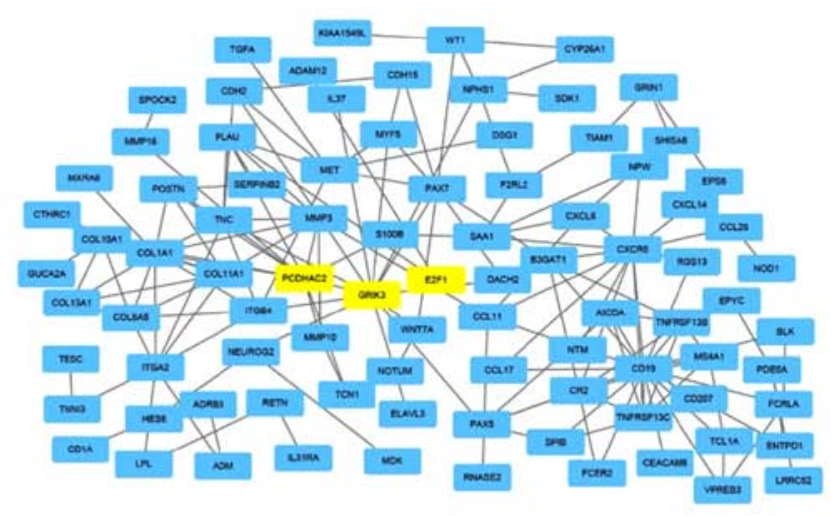

Blue module

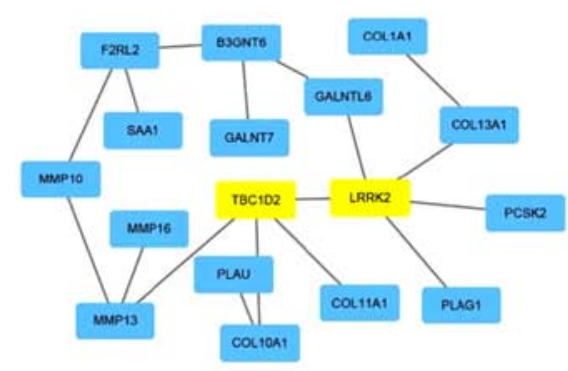

Yellow module

Figure 2. Protein-protein network visualization of different color modules by weighted gene co-expression network analysis. Each rounded rectangle corresponds to a protein-coding gene, while the yellow color indicated the key differentially expressed mRNAs at the core of modules.

hsa-mir-373, hsa-mir-144, hsa-mir-205, hsa-mir-221, hsa-mir-222, hsa-mir-187, hsa-mir-375 and hsa-mir-184, were subsequently identified to be closely associated with the aforementioned 12 hub DEmRNAs. A total of 30 DElncRNAs 
that were also closely associated with the DEmiRNAs were identified using the miRcode database (Table III). The DEmiRNAs and lncRNAs in the ceRNA network are presented in Table IV. Two highly clustered DElncRNAs in the ceRNA network were identified, AC022148.1 and OPCML intronic transcript 1 (OPCML-IT1). AC022148.1 interacted with hsa-mir-372, hsa-mir-373, hsa-mir-144, hsa-mir-519d, hsa-mir-205, hsa-mir-221, hsa-mir-222 and hsa-mir-31. OPCML-IT1 interacted closely with $7 \mathrm{miRNAs}$, including hsa-mir-372, hsa-mir-373, hsa-mir-519d, hsa-mir-184, hsa-mir-205, hsa-mir-506 and hsa-mir-375. The ceRNA network is presented in Fig. 3.

Survival and correlation analysis for the hub DEmRNAs, DEmiRNAs and DElncRNAs. A total of 12 DEmRNAs, 12 DEmiRNAs and 30 DElncRNAs were identified as hub genes in the tumorigenesis of CCA. To explore the effect these RNAs had on the overall survival time, Kaplan-Meier curve analysis was used to obtain a prognostic signature. Survival analysis in Fig. 4 revealed that two key mRNAs, SALL3 and E2F1, were associated with the progression of CCA. High SALL3 mRNA expression, but low E2F1 expression were associated with poor prognosis. Additionally, low expression levels of hsa-mir-144 and hsa-mir-184 were also associated with poor prognosis. MIR181A2HG and OPCML-IT1 had positive effects on the overall survival time of patients with CCA. Pearson's correlation coefficient analysis revealed that OPCML-IT1 has a stronger correlation with SALL3 in Fig. 5.

\section{Discussion}

With a high mortality and an increasing incidence rate globally, the mechanisms underlying CCA are currently being investigated (1-3). Numerous studies have revealed that dysregulated miRNAs and lncRNAs may be involved in tumor progression (22-24). Previous studies have demonstrated that non-coding RNAs may serve an important role in various biological processes $(4,24-28)$. The ceRNA network has been implicated in a number of different types of cancer and may contribute to tumor progression and metastasis in triple negative breast cancer (25), papillary renal cell carcinoma (26) and gastric cancer (27). A previous study revealed that two key ceRNA clusters involved in cell-based functions may contribute to CCA (28). However, the specific IncRNAs involved in the overall survival time in CCA remain unclear. The current study therefore comprehensively integrated mRNA and miRNA data from TCGA and constructed a ceRNA network of IncRNA-miRNA-mRNA interactions to further explore the mechanism of IncRNAs in CCA.

In the current study, 30 DElncRNAs were identified as the hub lncRNAs which were involved in the ceRNA network. MIR181A2HG and OPCML-IT1 were revealed to be potential prognostic biomarkers for CCA. As illustrated in the ceRNA network, these two DElncRNAs had more interactions and were involved in regulating the target genes by competing for a common miRNA (mir-205).

lncRNAs are non-protein-coding transcripts consisting of $>200$ nucleotides and have been revealed to regulate cellular processes (29). MIR181A2HG is the host gene of MIR181A2, and its overexpression has been associated with several types
Table III. Thirty DElncRNAs interact with the 12 DEmiRNAs as retrieved from the miRcode database.

\begin{tabular}{|c|c|}
\hline $\operatorname{lncRNA}$ & miRNAs \\
\hline IGF2-AS & hsa-mir-519d \\
\hline LINC00302 & hsa-mir-31, hsa-mir-506 \\
\hline AC022148.1 & $\begin{array}{l}\text { hsa-mir-372, hsa-mir-373, hsa-mir-144, } \\
\text { hsa-mir-519d, hsa-mir-205, hsa-mir-221, } \\
\text { hsa-mir-222, hsa-mir-31 }\end{array}$ \\
\hline LINC00313 & $\begin{array}{l}\text { hsa-mir-372, hsa-mir-373, hsa-mir-187, } \\
\text { hsa-mir-205, hsa-mir-31, hsa-mir-375 }\end{array}$ \\
\hline AC004832.1 & hsa-mir-519d, hsa-mir-31, \\
\hline AC002511.1 & hsa-mir-519d \\
\hline AC006305.1 & $\begin{array}{l}\text { hsa-mir-519d, hsa-mir-221, hsa-mir-222, } \\
\text { hsa-mir-506, hsa-mir-375 }\end{array}$ \\
\hline AP000525.1 & hsa-mir-31 \\
\hline UCA1 & hsa-mir-184, hsa-mir-506 \\
\hline AC010336.2 & $\begin{array}{l}\text { hsa-mir-372, hsa-mir-373, hsa-mir-144, } \\
\text { hsa-mir-519d, hsa-mir-205, hsa-mir-31 }\end{array}$ \\
\hline CLDN10-AS1 & hsa-mir-221, hsa-mir-222 \\
\hline MIR181A2HG & hsa-mir-205 \\
\hline LINC00365 & hsa-mir-519d \\
\hline LINC00457 & hsa-mir-144 \\
\hline SFTA1P & hsa-mir-221, hsa-mir-222 \\
\hline LINC00423 & hsa-mir-31 \\
\hline HOTAIR & $\begin{array}{l}\text { hsa-mir-519d, hsa-mir-221, hsa-mir-222, } \\
\text { hsa-mir-506, hsa-mir-375 }\end{array}$ \\
\hline HCG22 & hsa-mir-31, hsa-mir-506 \\
\hline MIR4500HG & hsa-mir-144, hsa-mir-31 \\
\hline MIR205HG & $\begin{array}{l}\text { hsa-mir-205, hsa-mir-221, hsa-mir-222, } \\
\text { hsa-mir-31, hsa-mir-506 }\end{array}$ \\
\hline CYP1B1-AS1 & hsa-mir-205 \\
\hline LINC00460 & hsa-mir-222, hsa-mir-221 \\
\hline LINC00284 & hsa-mir-519d, hsa-mir-205, hsa-mir-506 \\
\hline AC068594.1 & hsa-mir-222, hsa-mir-221 \\
\hline AC011383.1 & hsa-mir-31 \\
\hline SYNPR-AS1 & hsa-mir-375 \\
\hline GDNF-AS1 & hsa-mir-187 \\
\hline OPCML-IT1 & $\begin{array}{l}\text { hsa-mir-372, hsa-mir-373, hsa-mir-519d, } \\
\text { hsa-mir-184, hsa-mir-205, hsa-mir-506, } \\
\text { hsa-mir-375, }\end{array}$ \\
\hline AC110619.1 & hsa-mir-184 \\
\hline AP001029.2 & hsa-mir-31 \\
\hline
\end{tabular}

DE, differentially expressed; lncRNA, long non-coding RNA; miRNA, microRNA.

of cancer. Xu et al (30) demonstrated that MIR181A2HG was downregulated by estrogen in breast cancer. A previous study demonstrated that MIR181A2HG was upregulated in elderly patients with breast cancer (31).

The current study revealed that high expression of MIR181A2HG may compete with one key DEmiRNA (mir-205) to mediate the expression of target genes. In 
Table IV. Specific miRNAs, lncRNAs and mRNAs in the competing endogenous RNA network.

A, miRNA

\begin{tabular}{|c|c|c|c|c|}
\hline Gene symbol & Gene ID & Expression change & $\log 2$ fold change & P-value \\
\hline hsa-mir-519d & ENSG00000207981 & Up & 4.74 & $3.73 \mathrm{E}-06$ \\
\hline hsa-mir-31 & ENSG00000199177 & Up & 2.45 & $8.69 \mathrm{E}-20$ \\
\hline hsa-mir-506 & ENSG00000207731 & Up & 2.03 & $7.24 \mathrm{E}-13$ \\
\hline hsa-mir-372 & ENSG00000199095 & Up & 3.46 & 4.82E-09 \\
\hline hsa-mir-373 & ENSG00000199143 & Up & 2.55 & $3.35 \mathrm{E}-05$ \\
\hline hsa-mir-144 & ENSG00000283819 & Down & -2.14 & $1.80 \mathrm{E}-48$ \\
\hline hsa-mir-205 & ENSG00000284485 & Up & 2.08 & $1.12 \mathrm{E}-07$ \\
\hline hsa-mir-221 & ENSG00000207870 & Up & 3.36 & $1.92 \mathrm{E}-60$ \\
\hline hsa-mir-222 & ENSG00000207725 & Up & 3.02 & $9.01 \mathrm{E}-57$ \\
\hline hsa-mir-187 & ENSG00000207797 & Up & 3.17 & $8.72 \mathrm{E}-20$ \\
\hline hsa-mir-375 & ENSG00000198973 & Up & 2.89 & $1.10 \mathrm{E}-23$ \\
\hline hsa-mir-184 & ENSG00000207695 & Up & 3.62 & $6.05 \mathrm{E}-12$ \\
\hline
\end{tabular}

$\mathrm{B}, \operatorname{lncRNA}$

\begin{tabular}{|c|c|c|c|c|}
\hline Gene symbol & Gene ID & Expression change & $\log 2$ fold change & P-value \\
\hline IGF2-AS & ENSG00000099869 & Up & 3.94 & $7.11 \mathrm{E}-08$ \\
\hline LINC00302 & ENSG00000176075 & Up & 3.36 & 8.92E-08 \\
\hline AC022148.1 & ENSG00000180458 & Up & 3.13 & $3.85 \mathrm{E}-28$ \\
\hline LINC00313 & ENSG00000185186 & Up & 2.16 & $1.53 \mathrm{E}-11$ \\
\hline AC004832.1 & ENSG00000181123 & Down & -2.19 & $3.22 \mathrm{E}-20$ \\
\hline AC002511.1 & ENSG00000232680 & Down & -2.29 & $7.96 \mathrm{E}-30$ \\
\hline AC006305.1 & ENSG00000206129 & Down & -2.62 & $2.57 \mathrm{E}-42$ \\
\hline AP000525.1 & ENSG00000272872 & $\mathrm{Up}$ & 2.52 & $5.41 \mathrm{E}-17$ \\
\hline UCA1 & ENSG00000214049 & $\mathrm{Up}$ & 3.48 & $6.85 \mathrm{E}-12$ \\
\hline AC010336.2 & ENSG00000260500 & Up & 2.33 & $2.16 \mathrm{E}-28$ \\
\hline CLDN10-AS1 & ENSG00000223392 & $\mathrm{Up}$ & 4.19 & $1.58 \mathrm{E}-13$ \\
\hline MIR181A2HG & ENSG00000224020 & Up & 2.12 & $2.23 \mathrm{E}-41$ \\
\hline LINC00365 & ENSG00000224511 & Up & 2.39 & $5.59 \mathrm{E}-14$ \\
\hline LINC00457 & ENSG00000225179 & Up & 2.87 & $6.46 \mathrm{E}-12$ \\
\hline SFTA1P & ENSG00000225383 & Up & 2.19 & 3.09E-19 \\
\hline LINC00423 & ENSG00000226968 & Up & 3.17 & $4.16 \mathrm{E}-21$ \\
\hline HOTAIR & ENSG00000228630 & Up & 2.41 & $2.64 \mathrm{E}-06$ \\
\hline HCG22 & ENSG00000228789 & $\mathrm{Up}$ & 2.76 & $3.18 \mathrm{E}-19$ \\
\hline MIR4500HG & ENSG00000228824 & Down & -2.48 & $7.14 \mathrm{E}-21$ \\
\hline MIR205HG & ENSG00000230937 & $\mathrm{Up}$ & 2.63 & $6.43 \mathrm{E}-10$ \\
\hline CYP1B1-AS1 & ENSG00000232973 & Up & 2.26 & $9.22 \mathrm{E}-35$ \\
\hline LINC00460 & ENSG00000233532 & Up & 3.61 & $3.66 \mathrm{E}-20$ \\
\hline LINC00284 & ENSG00000233725 & $\mathrm{Up}$ & 3.91 & $8.27 \mathrm{E}-30$ \\
\hline AC068594.1 & ENSG00000263718 & Up & 2.65 & $1.38 \mathrm{E}-37$ \\
\hline AC011383.1 & ENSG00000253852 & Up & 2.37 & $1.86 \mathrm{E}-26$ \\
\hline SYNPR-AS1 & ENSG00000241359 & Up & 2.66 & 4.38E-07 \\
\hline GDNF-AS1 & ENSG00000248587 & Up & 3.59 & $1.08 \mathrm{E}-12$ \\
\hline OPCML-IT1 & ENSG00000254896 & Up & 5.02 & $5.08 \mathrm{E}-14$ \\
\hline AC110619.1 & ENSG00000218416 & Up & 2.09 & $2.36 \mathrm{E}-22$ \\
\hline AP001029.2 & ENSG00000267199 & $\mathrm{Up}$ & 2.69 & $1.10 \mathrm{E}-35$ \\
\hline
\end{tabular}


Table IV. Continued.

C, mRNA

\begin{tabular}{lcccr}
\hline Gene symbol & \multicolumn{1}{c}{ Gene ID } & Expression change & log2 fold change & P-value \\
\hline LRRK2 & ENSG00000188906 & Up & 4.02 & $7.71 \mathrm{E}-51$ \\
TBC1D2 & ENSG00000095383 & Up & 2.01 & $7.90 \mathrm{E}-42$ \\
E2F1 & ENSG00000101412 & $\mathrm{Up}$ & 2.01 & $7.55 \mathrm{E}-40$ \\
SHISA6 & ENSG00000188803 & $\mathrm{Up}$ & 3.91 & $6.01 \mathrm{E}-28$ \\
CYP1B1 & ENSG00000138061 & $\mathrm{Up}$ & 3.09 & $1.35 \mathrm{E}-24$ \\
TMEM100 & ENSG00000166292 & $\mathrm{Up}$ & 2.82 & $2.19 \mathrm{E}-20$ \\
PCDHAC2 & ENSG00000243232 & $\mathrm{Up}$ & 2.16 & $2.48 \mathrm{E}-20$ \\
GRIK3 & ENSG00000163873 & $\mathrm{Up}$ & 2.84 & $1.52 \mathrm{E}-19$ \\
FOXQ1 & ENSG00000164379 & $\mathrm{Up}$ & 2.10 & $1.94 \mathrm{E}-19$ \\
PCDHA1 & ENSG00000204970 & $\mathrm{Up}$ & 2.03 & $1.02 \mathrm{E}-13$ \\
HOXC13 & ENSG00000123364 & $\mathrm{Up}$ & 2.94 & $1.53 \mathrm{E}-07$ \\
SALL3 & ENSG00000256463 & $\mathrm{Up}$ & 2.52 & $6.15 \mathrm{E}-06$ \\
\hline
\end{tabular}

miRNA, micro RNA; lncRNA, long non-coding RNA.

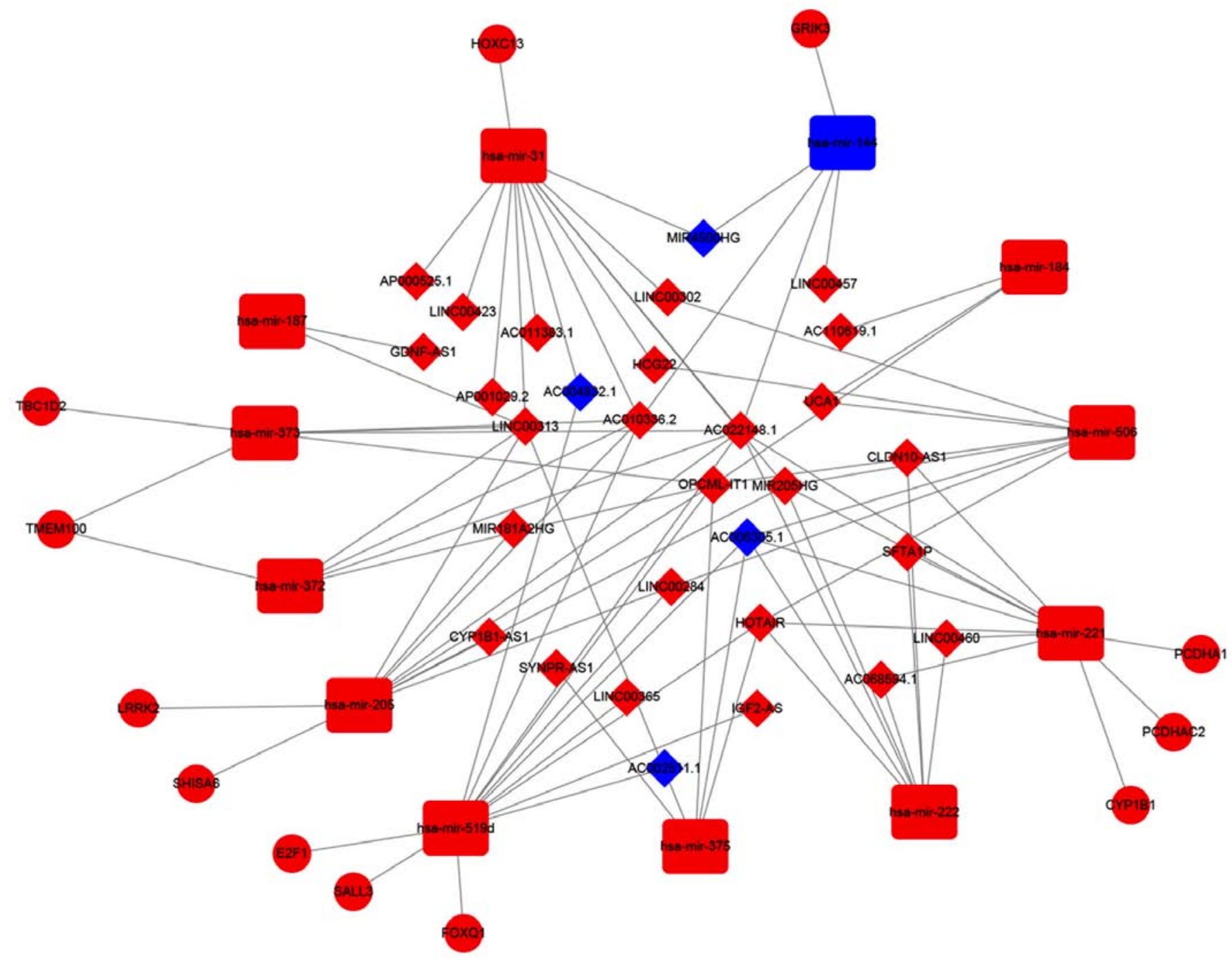

Figure 3. Identified competing endogenous RNA network in cholangiocarcinoma. The diamond, rounded rectangle and circle present long non-coding RNAs, microRNAs and mRNAs, respectively. Blue indicates downregulation while red indicates upregulation. 

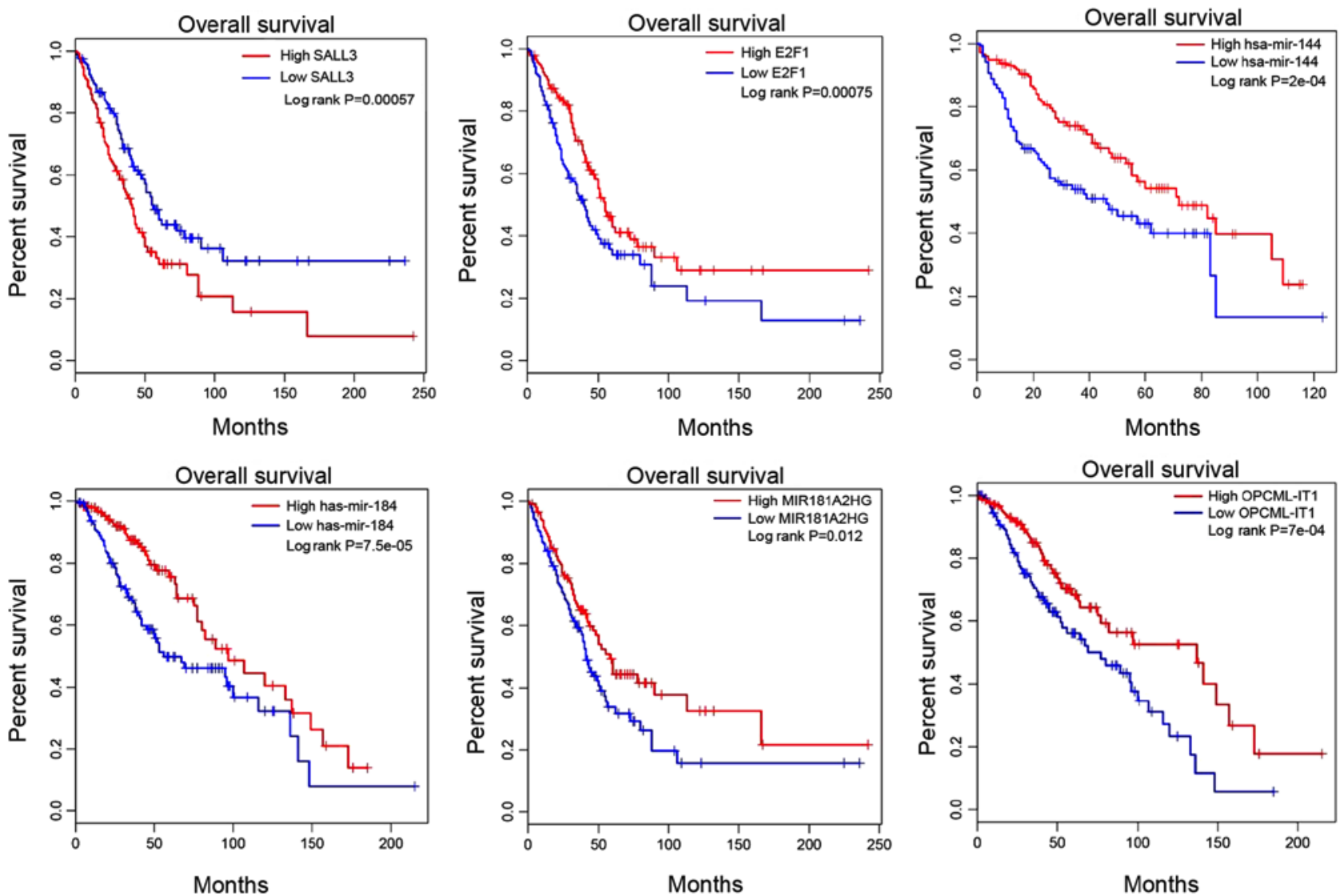

Figure 4. Survival analysis of DEmRNAs, DE microRNAs and DE long non-coding RNA. The horizontal axis presents the overall survival time, while the vertical axis presents the survival function. DE, differentially expressed; NC, negative control; C, control; lncRNA-NEF, long non-coding RNA-neighboring enhancer of FOXA2.

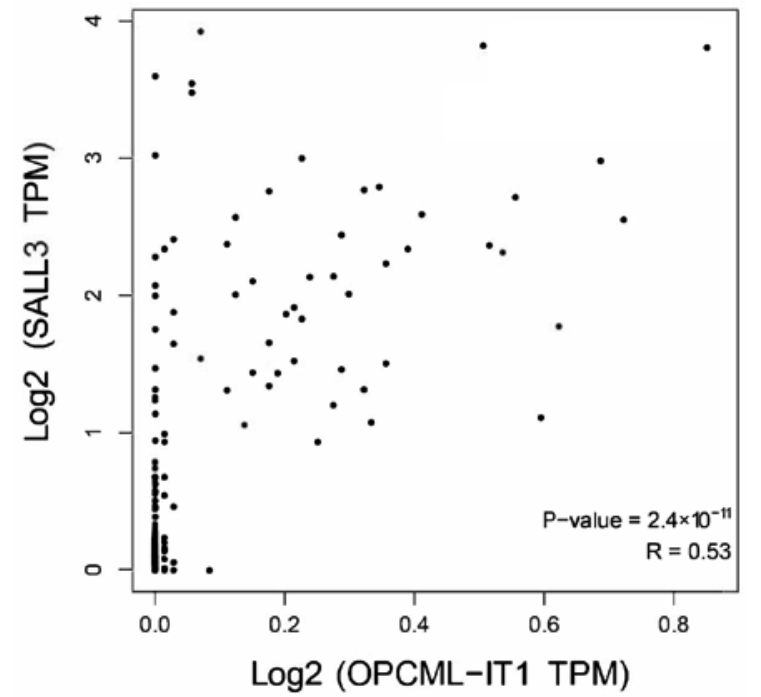

Figure 5. Pearson's correlation coefficient analysis between the expression levels of OPCML-IT1 and SALL3 in the competing endogenous RNA network. SALL3, spalt like transcription factor; OPCML-IT1, OPCML intronic transcript 1; TPM, transcripts per million.

addition, patients with highly expressed MIR181A2HG had shorter survival time compared with those with low expression according to the survival analysis performed in the current study. OPCML-IT1, another hub DElncRNA in the
ceRNA network, has been previously associated with gastric cancer (32). High expression of OPCML-IT1 may compete with seven key DEmiRNAs (mir-372, mir-373, mir-519d, mir-184, mir-205, mir-506 and mir-375) to mediate the expression of target genes. Furthermore, similar to MIR181A2HG, survival analysis revealed that increased expression of OPCML-IT1 was associated with a poor prognosis for patients with CCA. Based on the results of pathways analysis in the current study, knockdown of MIR181A2HG and OPCML-IT1 may prevent cell proliferation in CCA and prolong survival time of patients with CCA. miRNAs are master regulators of fine-tuning gene expression in a number of pathways, including cell cycle pathways (33). The current study demonstrated that 12 DEmiRNAs were involved in the ceRNA network, two of which (mir-144 and mir-184) were associated with differences in the overall survival time of patients with CCA. A previous study revealed that mir-144 may be an essential suppresser of CCA cell proliferation and invasion by targeting platelet activating factor acetylhydrolase $1 \mathrm{~b}$ regulatory subunit 1 (34). The survival analysis of mir-144 performed in the current study demonstrated that low expression of mir-144 can prolong patient survival. While mir-184 was revealed to be a target gene in breast (35) and liver (32) cancer, few studies have investigated the role of mir-184 in CCA $(35,36)$. Survival analysis indicated that high expression of mir-184 was associated with poor prognosis in patients with CCA. Thus, the mechanism of mir-184 in CCA requires further investigation. 
Based on the results of WGCNA performed in the current study, two protein-coding genes involved in the ceRNA network from four modules were identified, of which SALL3 and E2F1 were demonstrated to affect the overall survival time of patients with CCA. Epigenetic silencing of SALL3 was a predictor of poor survival in head and neck cancer (37). E2F1 is involved in the control of the cell cycle, proliferation and cell death (38). E2F1 was demonstrated to affect the overall survival in patients with prostate (39) and colorectal cancer (40). In the construction of ceRNA network, SALL3 or E2F1 can be a direct target of the hub DEmiRNA, mir-519d. Therefore, this DEmiRNA may compete with DElncRNA OPCML-IT1. Furthermore, the results of the regression analysis indicated a stronger correlation between SALL3 and OPCML-IT1 than between E2F1 and OPCML-IT1. Consequently, OPCML-IT1 may exhibit tumor-suppressing effects in CCA.

The current study had certain advantages over a previously published study (28): i) WGCNA was used to confirm the hub genes in functional modules; ii) the survival analysis of genes instead of clusters was investigated, which provided a clearer direction for future research; and iii) Pearson's correlation analysis was performed to validate the correlations between DElncRNAs and DEmRNAs involved in the formulated ceRNA network. Due to the several differences in the types of RNA-Seq gene expression, methods used for gene selection and cut-off criteria used for analyzing DEGs, there was no overlap between the current study and a previous study by Wan et al (28). The current study demonstrated the importance of SALL3 and OPCML-IT1 expression in patients with CCA, based on analysis of a weighted gene co-expression network, a ceRNA network and patient overall survival time. Future studies are required to verify the involvement of the ceRNA network in CCA.

\section{Acknowledgements}

Not applicable.

\section{Funding}

No funding was received.

\section{Availability of data and materials}

The datasets used and/or analyzed during the present study are available from the corresponding author on reasonable request.

\section{Authors' contributions}

YH, ZM, CH and XF conceived and designed the study. CL, PS, ZP, TY and MS collected the data and performed statistical analysis. ZM, CH and XF completed the manuscript.

\section{Ethics approval and consent to participate}

Not applicable.

\section{Patient consent for publication}

Not applicable.

\section{Competing interests}

The authors declare that they have no competing interests.

\section{References}

1. Banales JM, Cardinale V, Carpino G, Marzioni M, Andersen JB, Invernizzi P, Lind GE, Folseraas T, Forbes SJ, Fouassier L, et al: Expert consensus document: Cholangiocarcinoma: Current knowledge and future perspectives consensus statement from the European Network for the Study of Cholangiocarcinoma (ENS-CCA). Nat Rev Gastroenterol Hepatol 13: 261-280, 2016.

2. Jarnagin WR, Fong Y, DeMatteo RP, Gonen M, Burke EC, Bodniewicz BS J, Youssef BA M, Klimstra D and Blumgart LH: Staging, resectability, and outcome in 225 patients with hilar cholangiocarcinoma. Ann Surg 234: 507-519, 2001.

3. Maithel SK, Gamblin TC, Kamel I, Corona-Villalobos CP, Thomas M and Pawlik TM: Multidisciplinary approaches to intrahepatic cholangiocarcinoma. Cancer 119: 3929-3942, 2013.

4. Baltimore D: Our genome unveiled. Nature 409: 814-816, 2001.

5. Gong Z, Zhang S, Zeng Z, Wu H, Yang Q, Xiong F, Shi L, Yang J, Zhang W, Zhou Y, et al: LOC401317, a p53-regulated long non-coding RNA, inhibits cell proliferation and induces apoptosis in the nasopharyngeal carcinoma cell line HNE2. PLoS One 9: e110674, 2014.

6. Zhang W, Huang C, Gong Z, Zhao Y, Tang K, Li X, Fan S, Shi L, Li X, Zhang P, et al: Expression of LINC00312, a long intergenic non-coding RNA, is negatively correlated with tumor size but positively correlated with lymph node metastasis in nasopharyngeal carcinoma. J Mol Histol 44: 545-554, 2013.

7. Salmena L, Poliseno L, Tay Y, Kats L and Pandolfi PP: A ceRNA hypothesis: The Rosetta Stone of a hidden RNA language? Cell 146: 353-358, 2011.

8. Jeyapalan Z, Deng Z, Shatseva T, Fang L, He C and Yang BB: Expression of CD44 3'-untranslated region regulates endogenous microRNA functions in tumorigenesis and angiogenesis. Nucleic Acids Res 39: 3026-3041, 2011.

9. Poliseno L, Salmena L, Zhang J, Carver B, Haveman WJ and Pandolfi PP: A coding-independent function of gene and pseudogene mRNAs regulates tumour biology. Nature 465: 1033-1038, 2010.

10. Hu Q, Yin J, Zeng A, Jin X, Zhang Z, Yan W and You Y: H19 functions as a competing endogenous RNA to regulate EMT by sponging miR-130a-3p in glioma. Cell Physiol Biochem 50: 233-245, 2018

11. Zhao G, Fu Y, Su Z and Wu R: How long non-coding RNAs and microRNAs mediate the endogenous RNA network of head and neck squamous cell carcinoma: A comprehensive analysis. Cell Physiol Biochem 50: 332-341, 2018.

12. Liu XH, Sun M, Nie FQ, Ge YB, Zhang EB, Yin DD, Kong R, $\mathrm{Xia} \mathrm{R}, \mathrm{Lu} \mathrm{KH}$, Li JH, et al: Lnc RNA HOTAIR functions as a competing endogenous RNA to regulate HER 2 expression by sponging miR-331-3p in gastric cancer. Mol Cancer 13: 92, 2014.

13. Song X, Cao G, Jing L, Lin S, Wang X, Zhang J, Wang M, Liu W and Lv C: Analysing the relationship between lncRNA and protein-coding gene and the role of lncRNA as ceRNA in pulmonary fibrosis. J Cell Mol Med 18: 991-1003, 2014.

14. Yu G, Yao W, Gumireddy K, Li A, Wang J, Xiao W, Chen K, Xiao H, Li H, Tang K, et al: Pseudogene PTENP1 functions as a competing endogenous RNA to suppress clear-cell renal cell carcinoma progression. Mol Cancer Ther 13: 3086-3097, 2014.

15. Xi X, Chu Y, Liu N, Wang Q, Yin Z, Lu Y and Chen Y: Joint bioinformatics analysis of underlying potiential functions of has-let-7b-5p and core genes in human glioma. J Transl Med 17: $129,2019$.

16. Ivliev AE, t Hoen PA and Sergeeva MG: Coexpression network analysis identifies transcriptional modules related to proastrocytic differentiation and sprouty signaling in glioma. Cancer Res 24: 10060-10070, 2010.

17. Langfelder P and Horvath S: Eigengene networks for studying the relationships between co-expression modules. BMC Syst Biol 1: 54, 2007.

18. Langfelder P and Horvath S: WGCNA: An R package for weighted correlation network analysis. BMC Bioinformatics 9: $559,2008$.

19. Zhang B and Horvath S: A general framework for weighted gene co-expression network analysis. Stat Appl Genet Mol Biol 4: Article17, 2005. 
20. Hsu SD, Tseng YT, Shrestha S, Lin YL, Khaleel A, Chou CH, Chu CF, Huang HY, Lin CM, Ho SY, et al: miRTarBase update 2014: An information resource for experimentally validated miRNA-target interactions. Nucleic Acids Res 42 (Database Issue): D78-D85, 2014.

21. Jeggari A, Marks DS and Larsson E: miRcode: A map of putative microRNA target sites in the long non-coding transcriptome. Bioinformatics 28: 2062-2063, 2012.

22. Li P, Dong M and Wang Z: Downregulation of TSPAN13 by miR-369-3p inhibits cell proliferation in papillary thyroid cancer (PTC). Bosn J Basic Med Sci: Aug 2, 2018 doi: 10.17305/bjbms.2018.2865 (Epub ahead of print).

23. Luo L, Xia L, Zha B, Zuo C, Deng D, Chen M, Hu L, He Y, Dai F, $\mathrm{Wu}$ J, et al: miR-335-5p targeting ICAM-1 inhibits invasion and metastasis of thyroid cancer cells. Biomed Pharmacother 106: 983-990, 2018.

24. Xia E, Bhandari A, Shen Y, Zhou X and Wang O: lncRNA LINC00673 induces proliferation, metastasis and epithelial-mesenchymal transition in thyroid carcinoma via Kruppel-like factor 2. Int J Oncol 53: 1927-1938, 2018.

25. Yuan N, Zhang G, Bie F, Ma M, Ma Y, Jiang X, Wang Y and Hao X: Integrative analysis of lncRNAs and miRNAs with coding RNAs associated with ceRNA crosstalk network in triple negative breast cancer. OncoTargets Therapy 10: 5883-5897, 2017.

26. Huang C, Yuan N, Wu L, Wang X, Dai J, Song P, Li F, Xu C and Zhao X: An integrated analysis for long noncoding RNAs and microRNAs with the mediated competing endogenous RNA network in papillary renal cell carcinoma. Onco Targets Therapy 10: 4037-4050, 2017.

27. Li F, Huang C, Li Q and Wu X: Construction and comprehensive analysis for dysregulated long non-coding RNA (lncRNA)associated competing endogenous RNA (ceRNA) network in gastric cancer. Med Sci Monit 24: 37-49, 2018.

28. Wan M, Zhang FM, Li ZL, Kang PC, Jiang PM, Wang YM, Wang ZD, Zhong XY, Li CL, Wang $\mathrm{H}$, et al: Identifying survival-associated ceRNA clusters in cholangiocarcinoma. Oncol Rep 36: 1542-1550, 2016.

29. Ponting CP, Oliver PL and Reik W: Evolution and functions of long noncoding RNAs. Cell 136: 629-641, 2009.

30. Xu S, Kong D, Chen Q, Ping Y and Pang D: Oncogenic long noncoding RNA landscape in breast cancer. Mol cancer 16: 129, 2017.

31. Brouwers B, Fumagalli D, Brohee S, Hatse S, Govaere O, Floris G, Van den Eynde K, Bareche Y, Schöffski P, Smeets A, et al: The footprint of the ageing stroma in older patients with breast cancer Breast Cancer Res 19: 78, 2017.
32. Kang C, Song JJ, Lee J and Kim MY: Epigenetics: An emerging player in gastric cancer. World J Gastroenterol 20: 6433-6447, 2014.

33. Adams BD, Kasinski AL and Slack FJ: Aberrant regulation and function of microRNAs in cancer. Curr Biol 24: R762-R776, 2014.

34. Yang R, Chen Y, Tang C, Li H, Wang B, Yan Q, Hu J and Zou S: MicroRNA-144 suppresses cholangiocarcinoma cell proliferation and invasion through targeting platelet activating factor acetylhydrolase isoform 1b. BMC Cancer 14: 917, 2014.

35. Phua YW, Nguyen A, Roden DL, Elsworth B, Deng N, Nikolic I, Yang J, Mcfarland A, Russell R, Kaplan W, et al: MicroRNA profiling of the pubertal mouse mammary gland identifies miR-184 as a candidate breast tumour suppressor gene. Breast Cancer Res 17: 83, 2015.

36. Wu GG, Li WH, He WG, Jiang N, Zhang GX, Chen W, Yang HF, Liu QL, Huang YN, Zhang L, et al: Mir-184 post-transcriptionally regulates SOX7 expression and promotes cell proliferation in human hepatocellular carcinoma. PLoS One 9: e88796, 2014.

37. Misawa K, Mochizuki D, Imai A, Misawa Y, Endo S, Mima M, Kawasaki H, Carey TE and Kanazawa T: Epigenetic silencing of SALL3 is an independent predictor of poor survival in head and neck cancer. Clin Epigenetics 9: 64, 2017.

38. Lin M, Liu Y, Ding X, Ke Q, Shi J, Ma Z, Gu H, Wang H, Zhang C, Yang C, et al: E2F1 transactivates IQGAP3 and promotes proliferation of hepatocellular carcinoma cells through IQGAP3-mediated PKC-alpha activation. Am J Cancer Res 9: 285-299, 2019.

39. Ren Z, Kang W, Wang L, Sun B, Ma J, Zheng C, Sun J, Tian Z, Yang $X$ and Xiao W: E2F1 renders prostate cancer cell resistant to ICAM-1 mediated antitumor immunity by NF- $\mathrm{kB}$ modulation. Mol Cancer 13: 84, 2014.

40. Yang S, Wu B, Sun H, Ji F, Sun T, Zhao Y and Zhou D: Interrupted E2F1-miR-34c-SCF negative feedback loop by hyper-methylation promotes colorectal cancer cell proliferation. Biosci Rep 36: e00293, 2016.

This work is licensed under a Creative Commons Attribution-NonCommercial-NoDerivatives 4.0 International (CC BY-NC-ND 4.0) License. 